\title{
Antithrombotic therapy for patients with total artificial hearts
}

\author{
Oksana Volod ${ }^{1}$, Lee D. Lam ${ }^{2}$, Francisco A. Arabia ${ }^{3}$ \\ ${ }^{1}$ Department of Pathology, ${ }^{2}$ Cedars Sinai Comprehensive Transplant Center, Cedars Sinai, Los Angeles, CA, USA; ${ }^{3}$ Advanced Heart Program, \\ Banner University Medical Group, Phoenix, AZ, USA \\ Correspondence to: Oksana Volod, MD. Department of Pathology and Laboratory Medicine, Cedars Sinai, 8700 Beverly Blvd, Room 4711, Los \\ Angeles, CA 90048, USA. Email: oksana.volod@cshs.org.
}

Submitted Oct 23, 2019. Accepted for publication Dec 09, 2019.

doi: $10.21037 /$ acs.2019.12.01

View this article at: http://dx.doi.org/10.21037/acs.2019.12.01

\section{Introduction}

Morbidity and mortality following implantation of a total artificial heart (TAH) are increased by two common yet potentially avoidable adverse events: thromboembolic cerebral and peripheral vascular events $(1,2)$ and postoperative hemorrhage (3). The etiology of these prothrombotic and hemorrhagic adverse events is complex. Following implantation of a TAH, an individualized hemostatic response to the implant occurs. Hemolysis and infection may also contribute to the hemostatic imbalance. Heparin-induced thrombocytopenia (HIT), a systemic immune-mediated hypercoagulable condition, may occur in some patients after implantation (4-7). Postoperative hemorrhagic complications, which occur in up to $62 \%$ of TAH recipients (most commonly in the mediastinum and thorax) can be the sequelae of cardiopulmonary bypass, antithrombotic therapy intended to reduce the risk thrombotic events, or both. We have previously demonstrated that mechanical circulatory support (MCS) devices, which are commonly implanted for short-term use in patients with cardiogenic shock as a bridge to more durable device implantation, almost universally result in significant loss of von Willebrand factor multimers. This loss is often associated with an increased bleeding risk in the perioperative period when MCS devices are removed and a TAH is implanted (8). An acquired von Willebrand factor deficiency is commonly seen following the implantation of left ventricular assist devices but less commonly observed with TAH.

The need to manage both ends of the hemostatic spectrum, which may undergo daily alterations following implantation, requires individualized antithrombotic therapy. The most commonly used regimen consists of combination therapy, which includes an anticoagulant, one or more antiplatelet drugs, and perhaps an agent to minimize device-induced hemolysis.

\section{Agents}

\section{Anticoagulant therapy}

The ideal level of anticoagulation should be individually tailored. Low-intensity intravenously administered unfractionated heparin (UFH) is the mainstay therapy for bridging patients on MCS devices. The optimal level of anticoagulation for low-intensity protocols has not been standardized, but target values of the heparin assay between 0.15 and $0.3 \mathrm{IU} / \mathrm{mL}$ appear to be safe for bridging when using low-intensity intravenously administered UFH. Patients who are heparin-resistant due to antithrombin deficiency may require additional therapy (antithrombin III) to achieve adequate anticoagulation with heparin.

For long-term anticoagulation, warfarin is the most frequently used agent. Direct oral anticoagulants (DOACs) have replaced warfarin for many indications such as stroke prevention in patients with atrial fibrillation, with one advantage being that, unlike with warfarin, the use of DOACs does not require routine coagulation monitoring or dose titration. Although a recent study in 21 patients with left ventricular assist devices showed promising results (9), the use of DOACs in patients with MCS devices has not been clearly established; large prospective multicenter studies are necessary to confirm these findings.

When blood comes into contact with medical devices, such as TAHs, a coagulation cascade is triggered via 
intrinsic system pathways that are initiated by contact activation of factor XII. This is promulgated by subsequent activation of factor XI and ends in thrombin generation. We believe that factor XI and XII have a promising potential to be more targeted, and safer anticoagulants for use in patients with MCS devices. The factor XI-directed antisense oligonucleotide IONIS-416858 is the first agent that targets the contact pathway and is currently being tested in humans (10).

\section{Antiplatelet therapy}

Given the role of arachidonic acid in initiating and amplifying platelet activation, especially in a proinflammatory environment, aspirin is usually the standard antiplatelet agent for patients on MCS.

\section{Monitoring}

\section{Anticoagulant therapy}

The presence of lupus anticoagulant and concurrent warfarin administration, as well as high fibrinogen or factor VIII levels, may affect the activated partial thromboplastin time (aPTT), causing overestimation or underestimation of the heparin dose. Therefore, many institutions have recently switched UFH monitoring from aPTT to antifactor Xa assay (heparin assay). Because there is uncertainty whether the heparin assay is superior to aPTT in patients with MCS devices, we retrospectively reviewed patient records at our institution and discovered that $37 \%$ of our patients who were either undergoing MCS candidacy evaluation or were on an MCS device had positive lupus anticoagulant levels (unpublished data). Based upon this observation, the heparin assay appears to be more reliable than aPTT in patients with MCS devices but results from large multicenter studies using different instruments and reagents are necessary to establish uniform evidence-based monitoring guidelines.

Concurrent use of aPTT and the heparin assay to guide heparin therapy may be misleading. We believe that heparin therapy can be successfully guided via the use of thromboelastography (TEG 5000 series). aPTT determines adequacy of coagulation factor levels or degree of their inhibition in plasma, whereas TEG is a functional assay that reflects the interaction of platelets and other cellular contributors with factors in whole blood and is a closer representation of in vivo processes.
In our institution, warfarin dosing is pharmacogenetically based. Based on our pilot study (11), we propose establishing an international normalized ratio (INR) range that is individualized and patient-specific via the use of the TEG coagulation index and an INR starting goal (2.0-3.0), with the target INR range being that which results in the patient being in a normocoagulable state (coagulation index $\leq 1.2)$.

\section{Antiplatelet therapy}

Unlike the normal population, which may not require continuous aspirin monitoring, patients implanted with MCS devices are subject to conditions that may cause daily fluctuations in platelet function, thereby requiring frequent assessment of their hemostatic condition. The gold standard for evaluating platelet function to optimize and individualize antiplatelet therapy has traditionally been the use of optical platelet aggregometry. The assay is based on the optical measurement of platelet aggregation in platelet-rich plasma. We believe that, because hemolysis is present in all patients with MCS devices, optical platelet aggregometry is unsuitable for monitoring aspirin therapy, as is VerifyNow (Instrumentation Laboratory, Bedford, Massachusetts, USA) which is based on the turbidimetric optical detection method. For the last seven years, we have been using TEG Platelet Mapping (TPM modification of TEG 5000 device) (Haemonetics, Braintree, Massachusetts, USA) to monitor and manage anticoagulation (unfractionated heparin and warfarin) and antiplatelet (aspirin and dipyridamole) therapies in patients on MCS devices (11).

\section{Conclusion}

A variety of anticoagulant and antiplatelet strategies exist to treat patients implanted with a TAH; however, uncertainty still exists among experts in the field regarding the optimal treatment regimen for patients and the best way to assess the adequacy of any given treatment regimen. The healthcare team involved with the management of patients with TAHs is usually multidisciplinary. We believe that, to achieve optimal results, all members of the multidisciplinary TAH team should follow a standardized yet individually tailored anticoagulation protocol with clear guidelines specific to antithrombotic management of patients with TAH. Laboratory assays that assess anticoagulation adequacy must be simple to interpret and have defined target parameters. 


\section{Acknowledgments}

None.

\section{Footnote}

Conflicts of Interest: Oksana Volod is a consultant for Haemonetics. Francisco A. Arabia is a consultant for SynCardia Systems Inc. Lee D. Lam has no conflicts of interest to declare.

\section{References}

1. Arabía FA, Cantor RS, Koehl DA, et al. Interagency registry for mechanically assisted circulatory support report on the total artificial heart. J Heart Lung Transplant 2018;37:1304-12.

2. Torregrossa G, Morhuis M, Varghese R et al. Results with Syncardia total artificial heart beyond 1 year. ASAIO J 2014;60:626-34.

3. Copeland JG, Smith RG, Arabia FA et al. Cardiac replacement with a total artificial heart as a bridge to transplantation. N Engl J Med 2004;351:859-67.

4. Copeland JG, Copeland H, Gustafson M, et al. Experience with more than 100 total artificial heart implants. J Thorac Cardiovasc Surg 2012;143:727-34.

Cite this article as: Volod O, Lam LD, Arabia FA. Antithrombotic therapy for patients with total artificial hearts. Ann Cardiothorac Surg 2020;9(2):110-112. doi: 10.21037/ acs.2019.12.01
5. Thompson LO, Loebe M, Noon GP. What price support? Ventricular assist device induced systemic response. ASAIO J 2003;49:518-26.

6. Heilmann C, Geisen U, et al. Haemolysis in patients with ventricular assist devices: major differences between systems. Eur J Cardiothorac Surg 2009;36:580-4.

7. Warkentin TE, Greinacher A, Koster A. Heparin-induced thrombocytopenia in patients with ventricular assist devices: are new prevention strategies required? Ann Thorac Surg 2009;87:1633-40.

8. Goldfarb M, Czer LS, Lam LD, et al. High molecular weight von Willebrand factor multimer loss and bleeding in patients with short-term mechanical circulatory support devices: a case series. J Extra Corpor Technol 2018;50:77-82.

9. Parikh V, Parikh U, Ramirez AM, et al. Novel oral anticoagulants in patients with continuous flow left ventricular assist devices. J Heart Lung Transplant 2019;38:S425.

10. Weitz JI, Fredenburgh JC. Factors XI and XII as targets for new anticoagulants. Front Med (Lausanne) 2017;4:19.

11. Volod O, Lam LD, et al. Role of thromboelastography platelet mapping and international normalized ratio in defining "normocoagulability" during anticoagulation for mechanical circulatory support devices: a pilot retrospective study. ASAIO J 2017;63:24-31. 